Pensamiento Crítico Vol. $18 \mathrm{~N}^{\circ}$ 1, pp. 63-79

\title{
Los criterios epistemológicos en la investigación económica
}

Victor Orozco Livia

\section{RESUMEN}

El capitalismo ha cambiado de forma con la globalización, al unísono con los progresos de las ciencias, la tecnología y la filosofía. Cambios que han generado crisis en los paradigmas económicos.

La teoría monetarista, oficial para muchos gobiernos, ha sido cuestionada por los hechos económicos, mostrando deficiencias que provienen desde sus pilares epistemológicos. Si la teoría monetarista afirma con sus criterios epistemológicos que la investigación es observación de fenómenos, tal descripción de imágenes de entes o acontecimientos impide al pensamiento llegar hasta las causas internas que dan lugar al comportamiento de los objetos de estudio. A ello se debe que la crisis financiera del 2008 no se resuelva aún a fines del 2012. Las medidas económicas monetaristas sólo inciden en la imagen o apariencia de la crisis, más no en el contenido.

La investigación científica requiere de criterios epistemológicos que permitan al proceso de investigación ir más allá de las apariencias del objeto de estudio y llegar hasta las propiedades internas causantes de la crisis. Solo conociendo la naturaleza interna de la crisis se puede revertiría.

Palabras clave: globalización, Filosofía, Epistemología, Paradigmas. 


\title{
Pensamiento Crítico Vol. I8. N I
}

\begin{abstract}
Capitalism has changed with globalization, together with the progress of science, technology and philosophy. The changes that have generated economic paradigms crisis.

Monetarist theory, many government official, has been challenged by the economic facts, showing deficiencies that come from their epistemological pillars. If monetarist theory asserts its epistemological criteria that research is observation of phenomena, such description picture prevents entities or events thought to reach the internal causes that lead to the behavior of the objects of study. This is why the 2008 financial crisis still not resolved by the end of 2012. Monetarist economic measures only affect the image or appearance of the crisis, but not in content.

Scientific research requires epistemological criteria that allow the research process to go beyond the appearances of the object of study and get to the internal properties caused the crisis. Only by knowing the inner nature of the crisis can reverse it.
\end{abstract}

Keywords: globalización, philosophy, epistemology, paradigms

\section{La epistemología y la investigación económica}

La filosofía está inmersa en todo proceso de investigación, sobre todo la filosofía epistemológica. Los resultados de la investigación dependen en gran parte de los principios filosóficos y de los criterios epistemológicos que conllevan las investigaciones.

La filosofía es raciocinio crítico e integral del mundo basado en la ciencia. Trata de arribar a conclusiones generales sobre el ser o el mundo real, llevando a cabo inferencias con conocimientos probados que le remite la ciencia. La epistemología que es un acápite de la filosofía, se ocupa de estudiar las relaciones entre el ser y el pensamiento para tratar de establecer el desarrollo del conocimiento y sus características.

Los principios filosóficos y los criterios epistemológicos impregnan todo el proceso de investigación a fin de que éste, pueda arribar a conocimientos científicos

El procedimiento metodológico de la investigación conlleva conclusiones de razonamientos filosóficos y epistemológicos, desde el inicio hasta el final. A esto se debe que en el ámbito de los estudios económicos existan diversas corrientes de pensamiento económico, que hasta divergen totalmente los unos de los otros. De esta complejidad filosófica, epistemológica y procedimiento técnico en la investigación se 


\section{Victor Orozco Livia}

ocupa la metodología de la investigación, revelando en última instancia su postura filosófica. Y en efecto la filosofía hace metodología de investigación tanto para las ciencias naturales como para las ciencias sociales La pretensión es concluir en conocimientos científicos. Importa por consiguiente todo lo referente al conocimiento científico, que es materia de estudio por parte de la filosofía epistemológica A ello se debe que en estos tiempos se requiera saber con mayores precisiones que es el conocimiento científico y cuáles son sus cualidades de reconocimiento, considerando que se vive hoy en día una situación de crisis de paradigmas económicos. Pareciera que es una crisis estrictamente epistemológica tal como lo, plantea el economista Oswaldo de Rivero "Hace poco los premios nobeles Paul Krugman, Joseph Stiglitz, Robert Solow, George Akelof han admitido, en sendos artículos, que la economía es una disciplina que tiene serios problemas epistemológicos para enfrentar la realidad y predecir situaciones. Y que en consecuencia necesita una revisión de sus fundamentos" (Diario La Primera, 22 de setiembre de 2009.).

La ciencia, la filosofía y la epistemología, importan mucho por cierto para dilucidar las causas de la crisis de paradigmas económicos hoy en día, pero debe recordarse que primero es necesario saber de que ser social se trata. Porque resulta que este ser social que incluye la actividad económica de la humanidad, y del que se ocupa la ciencia social y la filosofía, ha cambiado de forma en las ultimas cuatro décadas. La última revolución científica-tecnológica ha generado cambios muy perceptibles en el comportamiento del ser social. Son cambios que se notan en lo tecnológico, económico, social, político, etc., y que ha dado lugar a la renovación de las ciencias y al progreso de la filosofía y los criterios epistemológicos, que pueden contar estos últimos con definiciones de mayor precisión respecto a todo lo referente al conocimiento científico. La metodología de investigación ha progresado mucho en las últimas décadas, a pesar de ser una materia nueva. Esto se debe al progreso de las ciencias que nutren a la filosofía para sus razonamientos, y a su vez la filosofía encamina a las ciencias por nuevos derroteros de investigación en aras de las verdades objetivas.

\section{La nueva forma hegemónica del ser social; La Globalización}

La actividad del ser humano en el mundo constituye la actuación del ser social sobre la modelación del mundo. De estas actividades humanas las económicas constituyen las predominantes en los cambios del mundo. A través de la historia de la humanidad el ser 


\section{Pensamiento Crítico Vol. I8. Nº}

social ha transformado al mundo y lo ha conducido a las condiciones actuales, en donde el capitalismo ha presentado una nueva forma de desarrollo que es la globalización. La globalización se ha convertido en el modo hegemónico de conducción del mundo en la actualidad y no precisamente para elevar las condiciones de bienestar de la población mundial, sino únicamente para privilegiar con sus beneficios a una reducida parte de la población. Como dice Joseph Stiglitz, Premio Nobel de la Economía en el 2001, “...para muchos en el mundo subdesarrollado la globalización no ha cumplido con sus promesas de beneficio económico.

La creciente división entre los poseedores y los desposeídos ha dejado a una masa creciente en el Tercer Mundo sumida en las más abyecta pobreza y viviendo con menos de un dólar por día". ${ }^{1}$ La globalización como nueva forma económica y social, de hegemonía capitalista ha gestado a sí mismo nuevas ideas, sobre todo en los ámbitos correspondientes a la filosofía, la economía y en el terreno político.

\section{La Globalización}

La globalización es la última generación de desarrollo del capitalismo en las actuales circunstancias. Antes de la globalización hubo capitalismo de competencia entre pequeños y medianos empresarios y después competencia entre monopolios.

La globalización empezó a gestarse después de la Segunda Guerra Mundial con los progresos de la ciencia que dio lugar a la creación de una nueva tecnología, cuyas aplicaciones a la empresa permitió la reconstrucción industrial de los países que participaron en la guerra.

Las grandes empresas destinaron parte de sus utilidades en investigación para innovación. Asimismo los gobiernos de los países desarrollados en apoyo de las grandes empresas destinaron parte del presupuesto para investigación y tecnología en aras de fomentar un crecimiento económico. La investigación pública y la privada concurrieron a la irrupción de la Tercera Revolución Científica - Tecnológica.

Las empresas que hicieron reconversión de tecnología arrasaron en el mercado a las que se aferraron a la de tecnología mecánica.. Para no perecer muchas empresas

1 Stiglitz, J. "El malestar en la globalización”. Ed. Taurus. Madrid, 2002. Pág. 29

\section{6}




\section{Victor Orozco Livia}

se fusionaron, integraron, hicieron alianzas, etc., con las de tecnología cibernética. Aparecieron así los conglomerados multinacionales que con la nueva tecnología compiten entre sí por mayor espacio en el mercado internacional Son conglomerados que compiten no solo para disminuir costos y elevar la productividad, sino también para colocar sus productos e invertir en industrias extractivas de materias primas en los países no desarrollados. Estas grandes corporaciones multinacionales reciben el apoyo de sus gobiernos y tienden a incidir en la política de los países no desarrollados a través del Consenso de Washington. Es una política que se fundamenta en la Teoría Monetarista y en la filosofía Neopositivista para darle un cariz convencional a la política económica neoliberal.

La globalización y la política neoliberal han profundizado la desigualdad en el mundo. Esta desigualdad que provoca la globalización en el mundo se nota con mucha crudeza en la economía más poderosa del mundo, como es la de Estados Unidos, y que puede apreciarse en la distribución de los ingresos que se muestra en el cuadro siguiente:

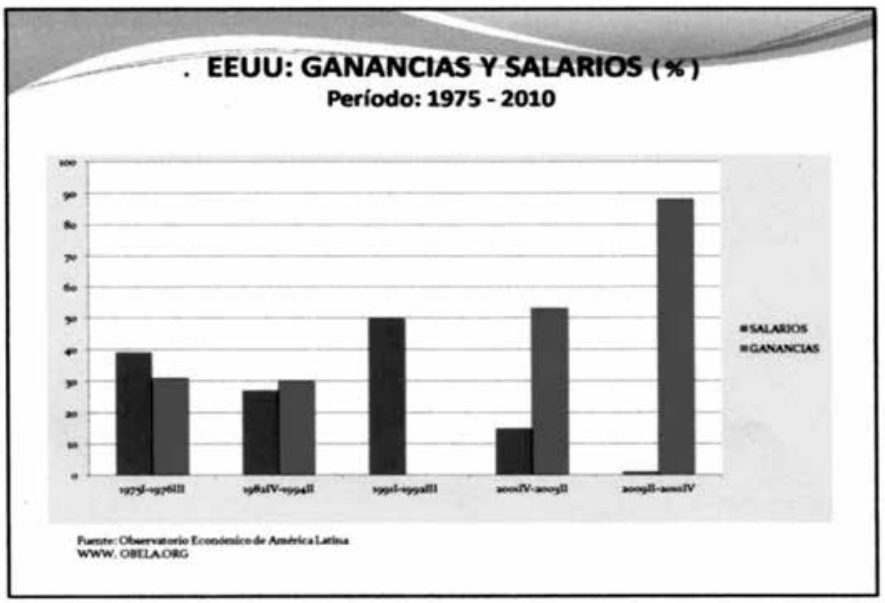

Fuente: Observatorio Económico de América Latina. / www.obela org

Contrastes socioeconómicos que han dado lugar a diversos movimientos sociales y culturales de inconformidad como los antiautoritarismos, la defensa de los derechos humanos." el feminismo, el ecologismo y los recientes movimientos de los jóvenes sin empleo, denominados "indignados". 


\section{Pensamiento Crítico Vol. I8. Nº}

\section{La globalización y las crisis de los paradigmas económicos}

La globalización y las crisis económicas en globalización han terminado por desnudar las graves inconsistencias de las teorías económicas contemporáneas.

La globalización no sólo ha constituido un cambio en las facetas económicas, sociales y políticas del capitalismo, sino que también ha incidido fuertemente en la detracción del socialismo El cambio de estas realidades ha dado lugar a nuevas ideas, unas a favor de la globalización y otras en criticas a la globalización.

Las ideas pilares del mundo neoliberal acorde con la globalización son la Filosofía Neopositivista, la Teoría Monetarista y el Consenso de Washington

Desde que apareció la teoría cibernética y la respectiva reconversión por parte de las grandes empresas, empezó a generarse diversas crisis económicas en globalización, siendo la mayor de ellas la que se presentó en el 2008. Las crisis que antecedieron a esta última, tanto la de fines del siglo XX como las de inicios del presente siglo pudieron ser atenuadas de algún modo pero al fin y al cabo la persistencia de sus contradicciones dio lugar a la del 2008.

Las crisis económicas constituyen tan solo una fase en el desenvolvimiento cíclico del sistema capitalista. A las crisis económicas le siguen las fases de depresión, reanimación y auge, que desemboca inevitablemente en una nueva crisis económica $A$ ello se debe que en la historia del capitalismo se ha experimentado alrededor de 160 crisis económicas. En el siguiente gráfico se representan algunas de las últimas crisis en globalización y sus consecuencias en el empleo. 


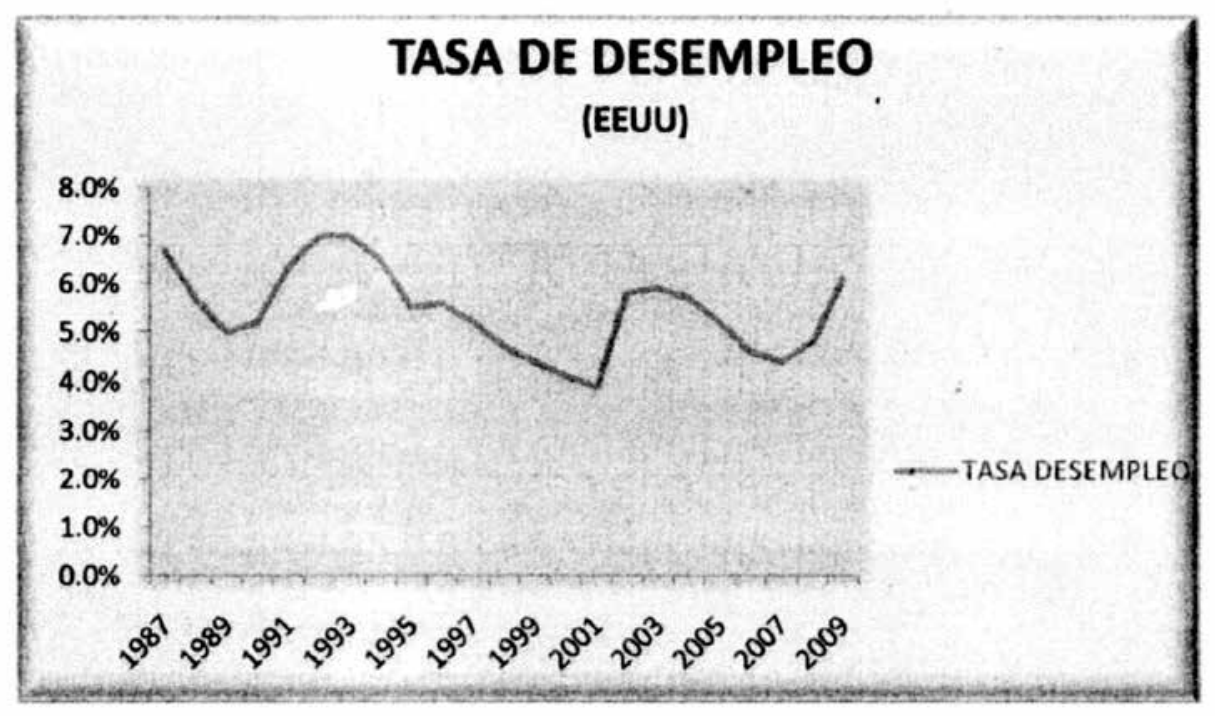

Fuente: Banco de St. Louis, Reserva Federal, 2008

\section{Oficina de Estadística del Mercado Laboral del Departamento de Trabajo Estados Unidos.}

Las últimas crisis económicas han ocurrido en épocas de globalización, escenario en el cual existe una tecnología muy perfeccionada como la cibernética, el directorio o accionistas de empresas son de conglomerados multinacionales, el capital financiero ha alcanzado grandes dimensiones, el obrero clásico de tecnología mecánica ha sido sustituido por trabajadores cognitarios, la producción y la comercialización de los productos y servicios abarca a una gran parte de países, que es terreno de disputa por mercado y concesiones de inversión en industrias extractivas y manufactureras.

A partir de 1970 fueron imponiéndose en el mercado las grandes empresas que incorporaron tecnología de punta, desplazando a las grandes empresas que conservaron tecnología mecánica. Las fusiones, integraciones o alianzas, con las empresas de tecnología moderna dan lugar al surgimiento de los conglomerados multinacionales. A medida que se consolidaban en el mercado internacional, las megaganancias que 


\section{Pensamiento Crítico Vol. I8. Nº}

obtuvieron estas nuevas corporaciones multinacionales crecieron enormemente, al punto de que sus depósitos en tos bancos rebasaron toda expectativa de éstos y a inicios del presente siglo había un gran problema de colocación que afectaba a los bancos. Desde entonces empezó a gestarse la gran crisis financiera del 2008, y que por inercia persisten sus efectos hasta los tiempos actuales. El exceso de liquidez en ios bancos con dificultad de colocación exigió una vulneración de las normas de regulación para el otorgamiento de créditos. Sobre todo la banca paralela que no se sujetaba al sistema de seguro de los depósitos Estas empezaron a otorgar créditos hipotecarios a solicitantes poco solventes. Al comienzo la inyección de estos créditos dinamizó al sector inmobiliario y los precios de las casas aumentaron, y cuando creció la morosidad por los prestamos inmobiliarios a insolventes, se desvalorizaron las cédulas hipotecarias, notándose en la Bolsa cotizaciones bajas y los precios de las inmobiliarias se vinieron abajo paralizando al sector construcción y creando un gran desempleo El siguiente cuadro estadístico ilustra la relación del Precio Vivienda-Renta Media y Precio AccionesIngresos Corporativos. Los bancos afectados por la morosidad, crearon activos con la garantía de las cédulas hipotecarias desvalorizadas y las difundieron en el mercado financiero internacional, expandiendo la crisis a la red bancada internacional y a las empresas vinculadas a ellas.

LAS BURBUJAS DE GREENSPAN

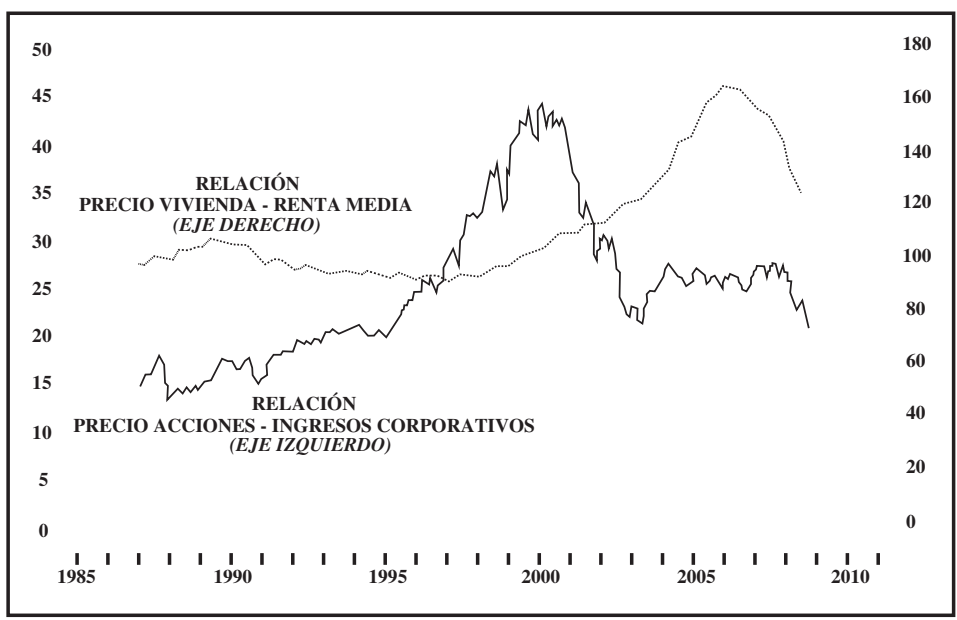

Fuente: Krugman, Paul. El Retorno de la Economía de la Depresión Ed. Letras de Crítica. Barcelona, 2009. Pág. 159 


\section{Victor Orozco Livia}

A partir del 2007 la saturación del sector inmobiliario, el descenso de los precios y el aumento del desempleo dio lugar a la crisis del 2008. Debe recordarse que en la economía actual todos los sectores de producción y de servicios están vinculados de un modo u otro, sobre todo por la red de créditos que los vincula. De modo que si uno de ellos ingresa a un proceso de estancamiento y crisis arrastra a los otros sectores. El incremento de morosidad en los bancos sobre todo por la insolvencia de las cédulas o bonos hipotecarios (subprimes), involucró al resto de los bancos importantes de Estados Unidos, a través del mercado financiero, incluidas la Bolsas de Valores y la crisis se hizo global porque el capital financiero hoy en día involucra a casi todos los bancos importantes del mundo.

El gobierno norteamericano trató de contener la crisis por medio de rescates financieros, es decir, prestamos a las entidades financieras y empresas importantes, primero con una inyección de 700,000 millones de dólares para el programa de rescate financiero y después con 800,000 millones de dólares adicionales, durante el gobierno de Obama. Los contribuyentes cargaron con el peso de la crisis porque el financiamiento se hizo con endeudamiento externo

En los países europeos también se recurrió a los rescates financieros, con préstamos al extranjero, elevando considerablemente la deuda externa de esos países, que obligó a estos gobiernos a recurrir a políticas de austeridad. Los casos más patéticos se vislumbran en Grecia y España. Pero estas intervenciones explícitas del Estado en la economía de los países no revirtió la crisis y sus ondas expansivas todavía están vigentes en Estados Unidos y Europa.

La Teoría Monetarista fue seriamente cuestionada por la realidad. Si uno de sus axiomas básicos era que el Estado no debe intervenir en la economía, los hechos le respondieron que aquello era ahora letra muerta. El descalabro de la Teoría Monetarista mostró ante la última crisis financiera sus graves deficiencias y por ello no se duda en los círculos académicos en reconocer que el paradigma monetarista esta en crisis.

El desarrollo de la globalización que es también expansión de los conglomerados multinacionales en muchas naciones del mundo, incidió también en las economías socialistas. 


\section{Pensamiento Crítico Vol. I8. Nº}

La tecnología cibernética de las grandes empresas capitalistas dejó rezagadas a las empresas del mundo socialista, ya que muchas de ellas todavía operaban con tecnología mecánica. La respuesta ante esta desigual competencia en el mercado internacional fue en un primer momento la apertura de sus mercados a las inversiones extranjeras como en Rusia, en otros casos como en Cuba la permisión progresiva del mercado. Tal vez las alternativas de China corno la de permitir inversiones extranjeras en su territorio con la condición de apropiación por parte del Estado de la tecnología que conllevaban estas inversiones, privatización masiva de empresas públicas, reducción de la tasa de interés y aumento del gasto publico, etc., obtuvo grandes resultados. Con tecnología de punta, salarios bajos y otorgamiento de mayor espacio al desarrollo del mercado. China conserva un crecimiento económico sostenido, sin purezas de socialismo El paradigma de la teoría economía socialista ante los hechos quedó con una nueva denominación Economía Socialista del Mercado, quedando en cuestión o en crisis el paradigma de la Teoría Económica Socialista.

\section{Consideraciones actuales para una metodología de investigación}

En el mundo intelectual de la ciencia económica se acepta que se vive una época de crisis de paradigmas económicos.

La nueva tecnología, la nueva forma del capitalismo, el progreso de las ciencias y la filosofía, qué cuenta esta ultima con diversas formas de razonamiento, entre otros factores, propugnan una metodología de investigación con mayores alcances, considerando que uno de sus soportes principales es la epistemología

La globalización ha suscitado grandes debates entre sus adherentes y sus críticos. Este intercambio de opiniones ha invadido y beneficiado el desarrollo de la Metodología de Investigación, la que ha cobrado mayor dimensión que la que le atribuían los metodólogos de las décadas pasadas.

En el terreno del razonamiento, por ejemplo, las neopositivistas siguen aferrados a al deducción de lógica formal, típica de la época medieval, mientras que la metodología contemporánea, considera diversos formas de razonar como son: La inducción y la deducción, el Análisis y la Síntesis, la teoría de Probabilidades, la Comparación y la 


\section{Victor Orozco Livia}

Analogía, la Teoría de Conjuntos, la Teoría de Juegos, el Raciocinio Cibernético, el Raciocinio Sistémico, etc., y deja abierta la posibilidad de emplear otras formas de razonar que puedan descubrirse en el futuro, siempre y cuando se utilicen según la rama científica y el tipo de investigación.

La Metodología de Investigación se ocupa de la investigación. Lo uno es lo teórico y lo otro lo práctico.

El ser humano investiga para establecer las causas del comportamiento de una realidad determinada, con lo cual se puede predecir su futuro comportamiento. En base a ello se elaboran medidas que tienden a la satisfacción de las necesidades humanas. En primer lugar, las columnas vertebrales de la metodología de la investigación científica en la actualidad son los principios filosóficos, los principios epistemológicos, la concepción científica del mundo del investigador y su ideología. En segundo lugar, los lineamientos de la investigación propiamente dicha, que abarca principalmente la formulación del problema, el marco teórico, el planteamiento de las hipótesis, la técnica de observación, los métodos racionales y la comprobación. Y en tercer lugar, está el método de exposición que es el discurso científico de los resultados de la investigación, que se elabora según principios, requisitos y respeto a las estructuras formales de exposición. La filosofía que compete a la Metodología de la Investigación Científica es la que se define como raciocinio crítico e integral del mundo, basado en la ciencia. Su objetivo es encontrar las regularidades que operan en el mundo o en grandes espacios con elementos similares.

La filosofía razona o demuestra racionalmente propiedades generales del mundo. La ciencia se ocupa de determinados aspectos del mundo y por consiguiente a descubrir leyes particulares del mundo. Con estas leyes particulares razona la filosofía para arribar a conclusiones generales sobre el mundo o sobre un conjunto de objetos de la misma clase.

La filosofía es raciocinio crítico, porque enfrentada a otras conclusiones que no emplean raciocinios a posteriori, ni emplea premisas basadas en la ciencia, puede demostrar que entre la filosofía sobre la realidad integral y lo que afirman las filosofías sin bases científicas, existe una disparidad, que no resiste a la critica filosófica. 


\section{Pensamiento Crítico Vol. I8. Nº}

La filosofía se ocupa del mundo o de grandes aspectos sectoriales del mundo con cualidades similares. Por ello es un raciocinio integral sobre el mundo o también filosofía sobre grandes aspectos importantes del mundo. Por ejemplo, una conclusión filosófica sobre el mundo biológico y social sería que en los entes biológicos y sociales se cumple la ley de la retroalimentación, lo que significa que en ciertos desvíos estructurales de la dirección en estos organismos, siempre existe capacidad de rectificación del rumbo de la dirección, por parte de sus propias fuerzas internas o con auxilio de los conocimientos humanos.

Los que hacen investigaciones biológicas o sociales deben tomar en cuenta esta ley, cuando se pretenda prescribir recomendaciones para controlar, regular o rectificar la dirección de algún fenómeno social o biológico. ${ }^{2}$

La filosofía además es la que hace metodología, tomando en cuenta los procedimientos de las ciencias particulares para generalizar sobre metodología en general y metodología para ciertas parcelas científicas particulares.

El otro aspecto importante ex ante de investigación es la dilucidación epistemológica. No se puede investigar si no se sabe que es el conocimiento. La epistemología aborda todo lo relacionado con el conocimiento. Por ejemplo, se ocupa del contenido del conocimiento y de su forma. El movimiento de aproximación infinito del pensamiento cognoscitivo a la realidad es el contenido del conocimiento y el discurso científico de lo descubierto en un determinado momento histórico es la forma del conocimiento. Por supuesto que la epistemología va mucho más allá de este tema sobre el conocimiento. Aborda asuntos como la relación entre el objeto de conocimiento y el sujeto de conocimiento para la generación de conocimientos, la fuente del conocimiento, el desarrollo del conocimiento, el método de conocimiento, el criterio de veracidad del conocimiento, etc. Conocer epistemología para investigar y conocer epistemología para escribir sobre lo investigado, es un requisito ex ante de investigación, contemplado por la Metodología de Investigación.

Existen otros tópicos ex ante de investigación que contempla la metodología y que contribuyen decididamente a los resultados de la investigación. Así por ejemplo, está la concepción científica del mundo del investigador, que por una parte es el conjunto de 2 Landa, N.L. Cibernética y Pedagogía. Biblioteca Universitaria Labor. Barcelona, 1972. Pág.19 


\section{Victor Orozco Livia}

teorías por las que opta el investigador para una visión total del mundo, y por otra parte es asimilación y comprensión del desarrollo y progreso de la ciencia ${ }^{3}$, que impregnan de uno u otro modo el resultado de la investigación. También está la ideología, que desempeña un papel importante en la investigación, si es acorde con las leyes generales del desarrollo de la humanidad expuestas en las teorías científicas, y si existe además una comprensión de derechos y responsabilidades cívicas en la que está situado el investigador. ${ }^{4}$

Para la metodología el proceso mismo de la investigación que es operativo, abarca una serie de fases que pueden ser agrupadas en tres momentos secuenciales. Se inicia con la detección de una realidad desconocida o no explicada por la ciencia, que constituye el problema de investigación. El siguiente momento es la elaboración de un proyecto de investigación para encarar lo desconocido y programar actividades que tiendan a dilucidar el problema y dejarlo apto para ser regulado o controlado. Es un proyecto en el que se programa lo que se va a investigar del problema, con que teoría referencial se va a contar, que propósitos se persigue en la investigación, cuales son las presunciones científicas de comportamiento de lo investigado, con que técnica de observación se va a obtener la información y como se va a procesar estos datos para presentarlos, y finalmente con que método se va a "analizar" la información. El tercer momento secuencial corresponde a la ejecución del proyecto, que significa recabar y procesar la información, para ser sometida a un raciocinio de contrastación de hipótesis, fruto de! cual aparecen las conclusiones y frente a éstas las recomendaciones.

Después de la preparación ex ante del investigador y ejecutado el proyecto de investigación se requiere elaborar una exposición de lo descubierto o comunicar a la comunidad científica los resultados de la investigación, bajo diversas modalidades, como reportes de investigación, tesis, artículos científicos, ponencias, etc. ${ }^{5}$

El discurso científico debe ceñirse a determinados principios como por ejemplo ir de lo simple a lo complejo. Cumplir determinados requisitos como por ejemplo el de la consistencia, que es coherencia interna o sin contradicciones entre sus partes y coherencia externa con la teoría que sirvió de orientación en la investigación, entre otros

3 Bijovski, B. La Concepción Científica del Mundo. Editorial E.P. La Habana, 1965. Pág.30

4 Gonzales Mires, C. Ideología y Conocimiento. U.N.S.A Cuzco, 1981. Pág.10

5 Velásquez Fernández, A. Metodología de la Investigación Científica. Ed. San Marcos. Lima, 1999, Pág.257 


\section{Pensamiento Crítico Vol. I8. Nº}

requisitos. ${ }^{6}$

Y finalmente, la estructura formal de presentación constituye también un conjunto de normas que se debe respetar. El título acorde con el contenido de lo que se explica en el cuerpo de la exposición, la introducción que presenta sucintamente el aspecto central de lo tratado, el cuerpo del desarrollo de la exposición, que son demostraciones del tema en no menos de tres capítulos, presentación de las conclusiones y recomendaciones, la bibliografía pertinente, los anexos, apéndices, etc.

\section{Las filosofías que propugnan epistemología}

La epistemología es el razonamiento filosófico sobre el conocimiento. Se ocupa del como y porque el ser humano puede tener conocimientos sobre el mundo o determinados aspectos de la realidad. En las entrañas de la filosofía se gesta la epistemología. Y con criterios epistemológicos el investigador tiende a llevar a cabo el proceso de investigación.

Las corrientes filosóficas contemporáneas discurren entre dos grandes vertientes discrepantes. Cada una de ellas presenta algunas aristas que no hacen la diferencia con lo esencial del contenido de su vertiente principal.

Por un lado está la filosofía idealista que integra hoy en día al racionalismo critico con Popper como mayor representante, el empirismo lógico con Carnap como su principal figura, la auto designada "filosofía de la ciencia" con Nagel como el más caracterizado, el atomismo lógico de Russell, etc., y que en términos generales se les ubica en la posición de la filosofía neopositivista. La filosofía neopositivista considera que la filosofía es análisis lógico del lenguaje y con esta concepción de filosofía tiene una propuesta epistemológica. La otra vertiente filosófica es la que deriva de la filosofía materialista clásica, y que tiene como representantes contemporáneos a R. Garaudy, Adolfo Sánchez, Maurice Cornforth, J.R. Núñez Tenorio, C. Guardia Mayorga, etc. Es una filosofía que se define como raciocinio crítico e integral del mundo basada en las ciencias contemporáneas, para encontrar las regularidades que se dan en el mundo. Los criterios epistemológicos de esta filosofía tienden a estructurar una teoría del conocimiento.

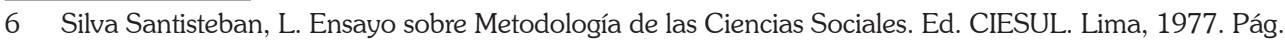




\section{Victor Orozco Livia}

Para comprender las diferencias epistemológicas de ambas escuelas filosóficas, presentaremos en primer lugar los criterios epistemológicos de la filosofía neopositivista.

Es una síntesis de lo propuesto por Karl Poper en su obra "La lógica de la Investigación Científica" lo expuesto por Mario Burge en "La Investigación Científica" y lo desarrollado por el economista Milton Friedman en su ensayo "Metodología de la Economía Positiva", que forma parte de su obra "Ensayos sobre Economía Positiva".

\section{La epistemología de la escuela filosófica neopositivista}

- El conocimiento, es la descripción del fenómeno o apariencias del objeto.

- El objeto de conocimiento, es el fenómeno de observación.

- El sujeto de conocimiento, es el que describe el fenómeno, sin ideas preconcebidas.

- La relación entre el objeto y el sujeto de conocimiento, es la que se da entre quién observa el fenómeno y el fenómeno en estudio.

- La fuente del conocimiento, es el conjunto de evidencias empíricas expresadas en palabras o signos con sentido.

- El desarrollo del conocimiento, es observación de un fenómeno con hipótesis descriptivas, evidencias empíricas y empleando lógica formal.

- El método de conocimiento, es el deductivo desligado de cualquier otro razonamiento conocido por la ciencia y la filosofía.

- El criterio de veracidad, es la validez del raciocinio deductivo según las formas de la lógica formal.

En seguida presentamos los criterios epistemológicos de la filosofía crítica basada en la ciencia, que constituye así mismo una apretada síntesis de lo que expresa A. M. Korshunov en "Actividad Conciencia y Conocimiento Científico", Fernando Salmaran 


\section{Pensamiento Crítico Vol. I8. N I}

en publicación del Instituto de Investigaciones Filosóficas de la UNAM y por último lo expresado por el filosofo peruano César Guardia Wayorga en su obra "Problemas del conocimiento".

- La epistemología propuesta por la filosofía basada en la ciencia.

- El conocimiento, es la actividad humana que tiende a representar la realidad en la conciencia. Procura trasponer las formas e ir al contenido.

- El objeto de conocimiento, es la realidad a la que se dirige el ser humano y su pensamiento para conocerla. Existe al margen de la conciencia humana

- El sujeto del conocimiento está conformado por el ser humano y su actividad cognoscitiva.

- La relación entre el objeto y el sujeto del conocimiento, es la actividad cognoscitiva del ser humano con el objeto de investigación.

- La fuente del conocimiento, es la realidad con sus características externas e internas que han de ser descubiertas por el pensamiento.

- El desarrollo del conocimiento, se constituye por aproximaciones sucesivas del pensamiento cognoscitivo a las interioridades de la realidad.

- El método de conocimiento, está constituido por los raciocinios aceptados por la ciencia y la filosofía a los que somete la información.

- El criterio de veracidad, se cumple en dos fases. Primero se razona y luego se comprueban las conclusiones en los hechos. Es la verdad objetiva

Con los principios de la filosofía crítica basada en la ciencia y sus correspondientes criterios epistemológicos la moderna metodología de Investigación adquiere la dimensión que a continuación se presenta en forma gráfica. 


\section{Propuestas de Metodología de Investigación}

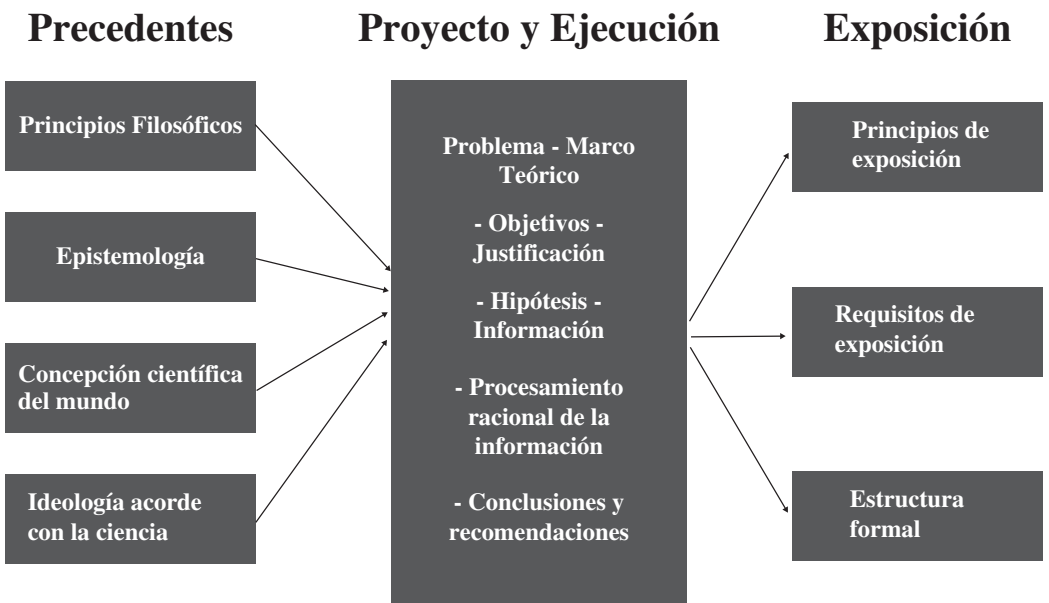

\section{BIBLIOGRAFÍA}

Reinert, Erik. La Globalización de la pobreza. Ed. Crítica Barcelona, 2007.

Stiglitz, Joseph. El malestar en globalización. Ed. Taurus. Madrid, 2002.

El retorno de la economía de la depresión. Ed. Letras de Crítica. Barcelona, 2009.

Marcet, José. iLlegó la crisis!. Ed. Granica. Barcelona, 2008.

Popper, Karl. La lógica de la Investigación científica. Ed. Estructura y función. Madrid, 1980.

Friedman, Milton. Ensayos sobre economía positiva. Ed. Gredos. Madrid, 1967.

Korshunov, A. M. Actividad, conciencia y conocimiento. Ed. Educap. Lima, 2006.

Maletta, Héctor. Metodología y Técnica de la Producción Científica. Ed. Universidad del Pacífico. Lima, 2009. 